\title{
Segmental order parameters in a nonionic surfactant lamellar phase studied with ${ }^{1} \mathrm{H}-{ }^{13} \mathrm{C}$ solid-state NMR
}

\author{
Tiago Mendes Ferreira, ${ }^{* a}$ Bruno Medronho, ${ }^{a b}$ Rachel W. Martin ${ }^{c}$ and Daniel \\ Topgaard*a
}

Received 6th May 2008, Accepted 2nd July 2008

First published as an Advance Article on the web 20th August 2008

DOI: $10.1039 / \mathbf{b 8 0 7 6 9 3 f}$

\begin{abstract}
A lyotropic nonionic lamellar system composed of pentaethyleneglycol mono $n$-dodecyl ether and $\mathrm{D}_{2} \mathrm{O}$ was studied using natural abundance ${ }^{13} \mathrm{C}$ NMR under magic-angle spinning. Applying a two-dimensional recoupling method proposed by Dvinskikh (R-PDLF), ${ }^{1} \mathrm{H}-{ }^{13} \mathrm{C}$ dipolar couplings were estimated over a range of temperatures (300-335 K), thus enabling analysis of structural changes in the liquid crystalline system. The results obtained are used to correlate the conformation and mobility of local sites in the surfactant molecule with overall changes in the lamellar structure.
\end{abstract}

\section{Introduction}

The relationship between the phase transitions of lyotropic systems such as oligo(oxyethylene) surfactants in aqueous solutions and the microscopic variations of the molecules, i.e., redistribution between conformational states due to changes in temperature and/or differences in concentration, has been the subject of a large number of studies. ${ }^{1}$ Over the last three decades, ${ }^{2} \mathrm{H}$ NMR has proven to be a very useful tool for this purpose. ${ }^{2-9}$ The measurement of the ${ }^{2} \mathrm{H}$ quadrupolar couplings provides an insight into the order profiles of C-D bonds and can reveal information about molecular orientation and motion in lipid membranes as well as other liquid crystalline systems.

The drawbacks of ${ }^{2} \mathrm{H}$ NMR spectroscopy methods result from the need for isotopic enrichment because of the low natural abundance $(0.01 \%)$ of deuterium. Although tedious selective labeling of specific segments can be avoided by using fully ${ }^{2} \mathrm{H}$ labeled samples, this is at the expense of resolution due to overlap of the different ${ }^{2} \mathrm{H}$ Pake patterns in the spectrum. Various NMR methods have been developed to obtain the ${ }^{13} \mathrm{C}-{ }^{1} \mathrm{H}$ dipolar couplings as an alternative to the more traditional ${ }^{2} \mathrm{H}$ NMR spectroscopy. ${ }^{10-16}$ These methods are less straightforward because the experiments are carried out under MAS. However, appropriate recoupling pulse sequences enable measurement of the heteronuclear dipolar couplings in an orthogonal dimension. This combines the high resolution of chemical sites provided by MAS with information about conformational and dynamical constraints. ${ }^{17,18}$

In this study, we probe a mixture of pentaethyleneglycol mono $n$-dodecyl ether (abbreviated as $\mathrm{C}_{12} \mathrm{E}_{5}$ ) and $\mathrm{D}_{2} \mathrm{O}$ (57 $\mathrm{wt}^{2} \% \mathrm{C}_{12} \mathrm{E}_{5}$ ), in the temperature range $300-335 \mathrm{~K}$, using

\footnotetext{
${ }^{a}$ Department of Physical Chemistry 1, Lund University, P.O. Box 124, SE-22100 Lund, Sweden. E-mail: Tiago.Ferreira@fkem1.lu.se; Daniel.Topgaard@fkem1.lu.se

${ }^{b}$ Departamento de Quimica, Universidade de Coimbra, 3004-535 Coimbra, Portugal

${ }^{c}$ Department of Chemistry, University of California, Irvine, CA 92697-2025, USA
}

a recoupling scheme proposed by Dvinskikh, called R-PDLF (R-type proton detected local field). ${ }^{16}$ This scheme was recently used to probe the segmental order of lipid membranes. ${ }^{19-21}$ Here, we apply the R-PDLF method for the first time to a surfactant system. According to the phase diagram reported by Imai et al. ${ }^{22}$ the temperature range studied is within the lamellar phase region.

Every 2D NMR experiment is based on assembling 1D free induction decays from increasing times $t_{1}$ in the indirect dimension. The modulation of the signal depends on the anisotropic interactions chosen during this time period. The Hamiltonian terms $\left(H_{\mathrm{CSA}}+H_{\mathrm{II}}+H_{\mathrm{IS}}\right) ; H_{\mathrm{CSA}}$, chemical shift anisotropy, $H_{\mathrm{II}}$, homonuclear dipolar coupling, $H_{\mathrm{IS}}$, heteronuclear dipolar coupling; can be activated or deactivated during $t_{1}$, depending on the information that one wants to get from the indirect dimension. ${ }^{23}$

When performing MAS NMR the $H_{\mathrm{CSA}}, H_{\mathrm{II}}$ and $H_{\mathrm{IS}}$ terms of the Hamiltonian are suppressed when the condition, spinning frequency $>$ interaction frequency is met. This means achieving high resolution spectra at the expense of valuable information. A wide range of recoupling methodology under MAS has been proposed in the last two decades to selectively restore the information of the heteronuclear dipolar couplings. ${ }^{13,15,18,24-28}$ In this study we use an R-type proton detected local field method (R-PDLF).

This experiment (Fig. 1) was proposed by Dvinskikh et al. ${ }^{16}$ and results from the incorporation of an RF scheme, R187, presented by Zhao et al., ${ }^{15}$ into a proton detected local field $(\mathrm{PDLF})^{11}$ protocol. The R18 $8_{1}^{7}$ scheme allows the recoupling of heteronuclear dipolar couplings and chemical shift anisotropies (CSAs) while homonuclear dipole couplings are suppressed under MAS. The ${ }^{1} \mathrm{H}$ CSAs can be deactivated by a ${ }^{13} \mathrm{C}$ $180^{\circ}$ pulse followed by a $180^{\circ}$ shift of the first $\mathrm{R} 18_{1}^{7}$ block phase. Each R18 1 block consists of a repeated sequence of $180^{\circ}{ }_{70} 180^{\circ}{ }_{290}$ pulses, each one with a length of $1 / 18$ of the rotor period. Increasing the time length $t_{1}$, modulates the effect of the dipolar field on each proton attached to a ${ }^{13} \mathrm{C}$ atom. This step is then followed by a polarization transfer from ${ }^{1} \mathrm{H}$ to

${ }^{13} \mathrm{C}$ using a rotor synchronized refocused INEPT (insensitive 


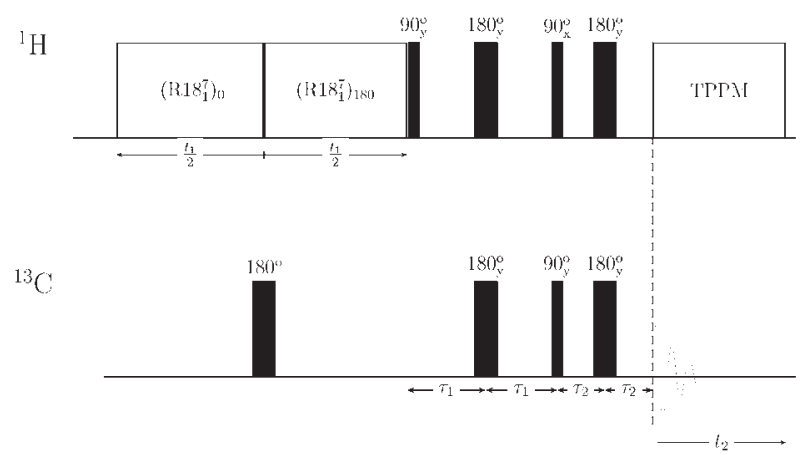

Fig. 1 Pulse sequences in the R-PDLF experiment. The dipolar couplings are activated during $t_{1}$ and modulate the amplitude of the isotropic chemical shifts during $t_{2}$.

nuclei enhanced by polarization transfer). ${ }^{29,30}$ After this transfer, the free induction decay of the ${ }^{13} \mathrm{C}$ nuclei is recorded while applying TPPM $^{31}$ decoupling of the protons. Performing Fourier transformation in both the direct and indirect dimensions, a 2D spectrum is obtained that correlates the ${ }^{13} \mathrm{C}$ chemical shifts and the heteronuclear dipolar couplings.

The advantage of the R-PDLF over other recoupling schemes is that it enables measurement of the average dipolar field that the protons of a ${ }^{13} \mathrm{C}-\mathrm{H}_{x}$ spin system experience from the rare ${ }^{13} \mathrm{C}$ nucleus during the time $t_{1}$. This is accomplished because the dipolar field that protons feel is most likely originated by a single ${ }^{13} \mathrm{C}$ nucleus (natural abundance of $1 \%$ ), and due to the selectivity of the INEPT transfer. The splitting shown by a ${ }^{13} \mathrm{C}-{ }^{1} \mathrm{H}$ spin pair in an R-PDLF experiment is given by $\Delta v_{\mathrm{CH}}=0.315 d_{\mathrm{CH}}$, where 0.315 is an effective scaling factor and $d_{\mathrm{CH}}$ is the ${ }^{13} \mathrm{C}^{-1} \mathrm{H}$ dipolar coupling. $d_{\mathrm{CH}}$ can be written as $d_{\mathrm{CH}}=b_{\mathrm{CH}}\left(3 \cos ^{2} \theta-1\right)$, with $b_{\mathrm{CH}}=-\left(\mu_{0} / 4 \pi\right) \times$ $\left(\gamma_{\mathrm{H}} \gamma_{\mathrm{C}} \hbar / r^{3}\right)$ as the ${ }^{13} \mathrm{C}-{ }^{1} \mathrm{H}$ dipolar coupling constant and $\theta$ as the angle between the internuclear vector and the magnetic field. In the case of rapid motion due to conformational changes and/or diffusion of the molecules, the splitting, $\Delta v$, is proportional to the segmental order parameter $S_{\mathrm{CH}}=\frac{1}{2}\left\langle 3 \cos ^{2} \theta-1\right\rangle$.

Temperature-dependent transitions from classical lamellar phases to random mesh phases or "porous" lamellar phases have been shown to exist in numerous studies. ${ }^{6,9,22}$ It has been argued that the fluctuations of order parameters with temperature in nonionic surfactant mixtures can be interpreted as a measure of perforation density. In this paper we interpret the temperature dependence of the dipolar couplings from a simple conformational basis.

\section{Experimental}

\subsection{Sample preparation}

Pentaethylene glycol dodecyl ether $\left(\mathrm{C}_{12} \mathrm{E}_{5}\right)$ with a purity higher than $99.8 \%$ was purchased from Nikko Chemical Co. (Tokyo, Japan). Deuterium oxide $\left(\mathrm{D}_{2} \mathrm{O}\right)$ was obtained from Sigma Chemicals (Steinheim, Germany). Samples containing $57 \mathrm{wt} \%$ surfactant were prepared by weighing the desired amounts of surfactant and water into vials, mixing them in a vortex mixer and centrifuging them in order to remove air bubbles.

\subsection{NMR measurements}

The experiments were performed using a Bruker Avance AVII-500 NMR spectrometer operating at a ${ }^{1} \mathrm{H}$ frequency of $500.23 \mathrm{MHz}$ equipped with a standard bore CP-MAS HX probe. All experiments were done at the spinning frequency of $5 \mathrm{kHz}$. The 2D NMR spectra were measured using the pulse sequence shown in Fig. 1 with the following parameters: spectral width of $100.4 \mathrm{ppm}, \tau_{1}=1.78 \mathrm{~ms}$ and $\tau_{2}=1.19 \mathrm{~ms}$ $\left(\tau_{1}=\frac{1}{4 J_{\mathrm{CH}}}\right.$ and $\tau_{2}=\frac{1}{6 J_{\mathrm{CH}}}$ with $\left.J_{\mathrm{CH}}=140 \mathrm{~Hz}\right)$, power levels were set to give the following nutation frequencies: $\mathrm{R} 18_{1}^{7}$ pulses $=45.00 \mathrm{kHz},{ }^{13} \mathrm{C}$ pulses $=63.45 \mathrm{kHz},{ }^{1} \mathrm{H}$ INEPT pulses $=63.45 \mathrm{kHz},{ }^{1} \mathrm{H}$ decoupling pulses $=25 \mathrm{kHz} . t_{1}$ increased up to $51.1488 \mathrm{~ms}$ taking values of $11.1 \mu \mathrm{s} \times m \times$ $18 \times 2$ with $m=1, \ldots, 64$, each R 187 block being composed of $18 \times m \pi$ pulses of length $11.1 \mu$ s. For each $t_{1}$ value, 32 scans were measured with a recycle delay of $4 \mathrm{~s}$. With a shorter delay, RF-heating could be detected via changes in chemical shift at the longest values of $t_{1}$. For each scan 10098 time domain points were acquired using a spectral width of $100.4 \mathrm{ppm}$. The experiments were performed at temperatures $335,330,326,323,320,317,314,310,305$ and $300 \mathrm{~K}$ in both increasing and decreasing directions. The temperature was calibrated using a methanol sample (see ref. 32 for more details). A 2D spectral processing scheme was applied. After Fourier transformation in both dimensions, each frequency domain signal in the indirect dimension was processed as follows. The zero frequency component in the indirect dimension was fixed as the value of the adjacent points. After inverse Fourier transform, sine-bell apodization and zero filling were performed on the time signal obtained. The frequency signal was recalculated and splittings measured. Because small splittings were obscured by the characteristic middle peak of the R-PDLF dipolar frequency signal (Fig. 2A), these were calculated directly from the time domain, using the expression, $\Delta v=2 / t_{\min }$, where $t_{\min }$ is the position of the minimum in the time signal (Fig. 2B).

\section{Results}

Fig. 3 shows the ${ }^{13} \mathrm{C}$ NMR spectrum of a $\mathrm{C}_{12} \mathrm{E}_{5} /$ water mixture with $57.7 \mathrm{wt} \% \mathrm{C}_{12} \mathrm{E}_{5}$ at $300 \mathrm{~K}$ using the R-PDLF method. The peak assignments were made on the basis of previous reports for $\mathrm{C}_{12} \mathrm{E}_{6}{ }^{33}$ and $\mathrm{C}_{12} \mathrm{E}_{8} \cdot{ }^{34}$ In the last one, it is shown that the highest band at approximately $70 \mathrm{ppm}$, corresponds to the interior chain of oxyethylene units (from the penultimate group to the most inner one) as the intensity of this band increases with increasing oxyethylene content. With respect to the assignment of the peaks to the carbons of the alkyl chain, $\mathrm{C}_{1}\left(\mathrm{C}_{\alpha}\right), \mathrm{C}_{9}, \mathrm{C}_{10}, \mathrm{C}_{11}$ and $\mathrm{C}_{12}$, all of these were assigned as in the previous publications. The peak at $37 \mathrm{ppm}$ that was previously assigned as being $\mathrm{C}_{2}$ was here assigned as $\mathrm{C}_{4}$. The new assignment was based on the assumption that the first derivative, $\partial \delta / \partial \mathrm{T}$, of the chemical shifts as a function of temperature should decrease from $\mathrm{C}_{1}$ to $\mathrm{C}_{4}$ (Fig. 4).

Fig. 5 shows a contour plot of the relevant regions of a $2 \mathrm{D}$ spectrum taken at $335 \mathrm{~K}$. The assignment of the partially overlapping peaks in both oxyethylene and alkyl carbon highest bands, 70 and 29 ppm, respectively, was based on 

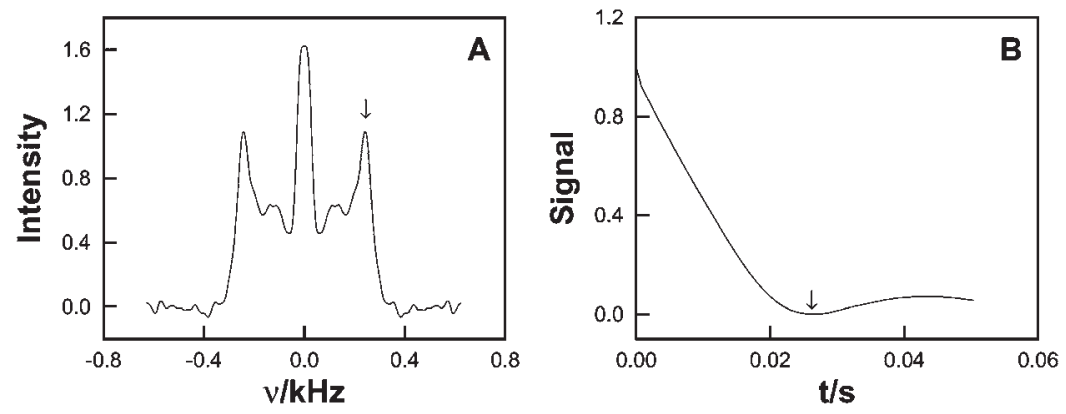

Fig. 2 (A) Frequency domain signal from $\mathrm{C}_{3}$ and (B) time domain signal from $\mathrm{C}_{12}$. The arrows show the maximum used to calculate splittings from the frequency domain, and the minimum used to calculate small splittings.
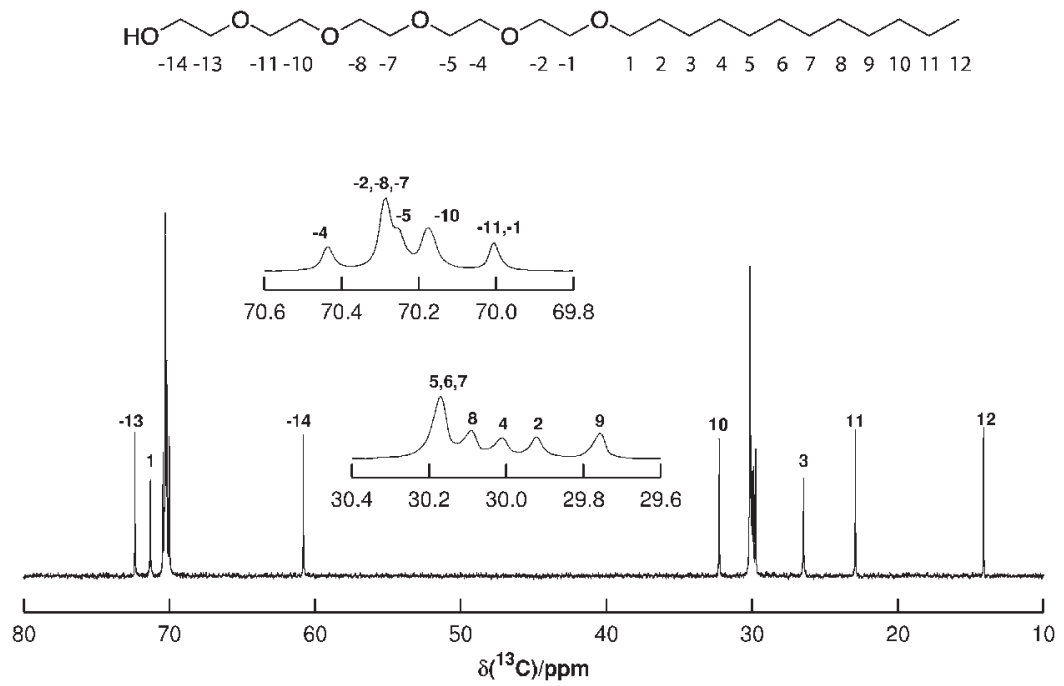

Fig. $3{ }^{13} \mathrm{C}$ NMR spectrum of a $\mathrm{C}_{12} \mathrm{E}_{5} / \mathrm{D}_{2} \mathrm{O}$ mixture with $57.7 \mathrm{wt} \% \mathrm{C}_{12} \mathrm{E}_{5}$ at $300 \mathrm{~K}$.

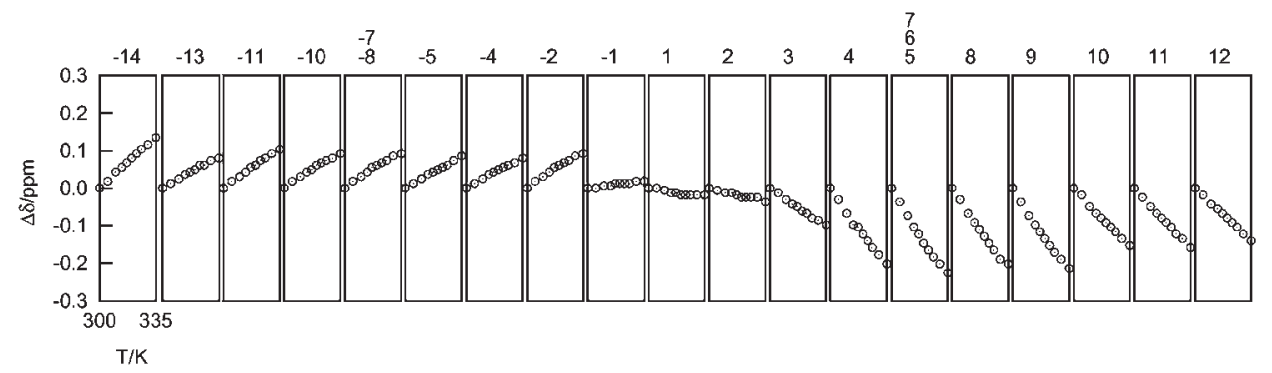

Fig. 4 Chemical shift dependence on temperature. $\Delta \delta=\delta-\delta_{300 \mathrm{~K}}$. The numbers on top identify the different carbons.

the assumption that the splittings decrease in the direction of the terminal groups in agreement with previous ${ }^{2} \mathrm{H}$ NMR experimental results. ${ }^{3,8}$

The cause of the ordering for $\mathrm{C}-\mathrm{H}$ bonds in the molecules can be split into three different classes (Fig. 6).

(I) MAS effects In static experiments, lamellar phases orient with the normal vector perpendicular to the magnetic field. ${ }^{35}$ Under MAS, mechanical forces from the spinning dominate, giving rise to different distributions in the orientation of the lamellae. Even at equal MAS frequency values, a different averaging between orientations may be expected depending on the viscosity of the sample.
(II) Lamellar phase morphology The curvature of the individual lamellae will have an effect on the average orientation of the molecules.

(III) Molecular mobility On the single molecule level, the conformational distribution along the $\mathrm{C}-\mathrm{C}$ and $\mathrm{C}-\mathrm{O}$ bonds will produce different average orientations with respect to each ${ }^{1} \mathrm{H}-{ }^{13} \mathrm{C}-{ }^{1} \mathrm{H}$ spin system.

The effects described in I and II are unlikely to change much with temperature, provided the temperature change is not large enough to cause a phase transition, and they should produce a similar trend on the order parameters of the different segments. Therefore, comparison of the change in 

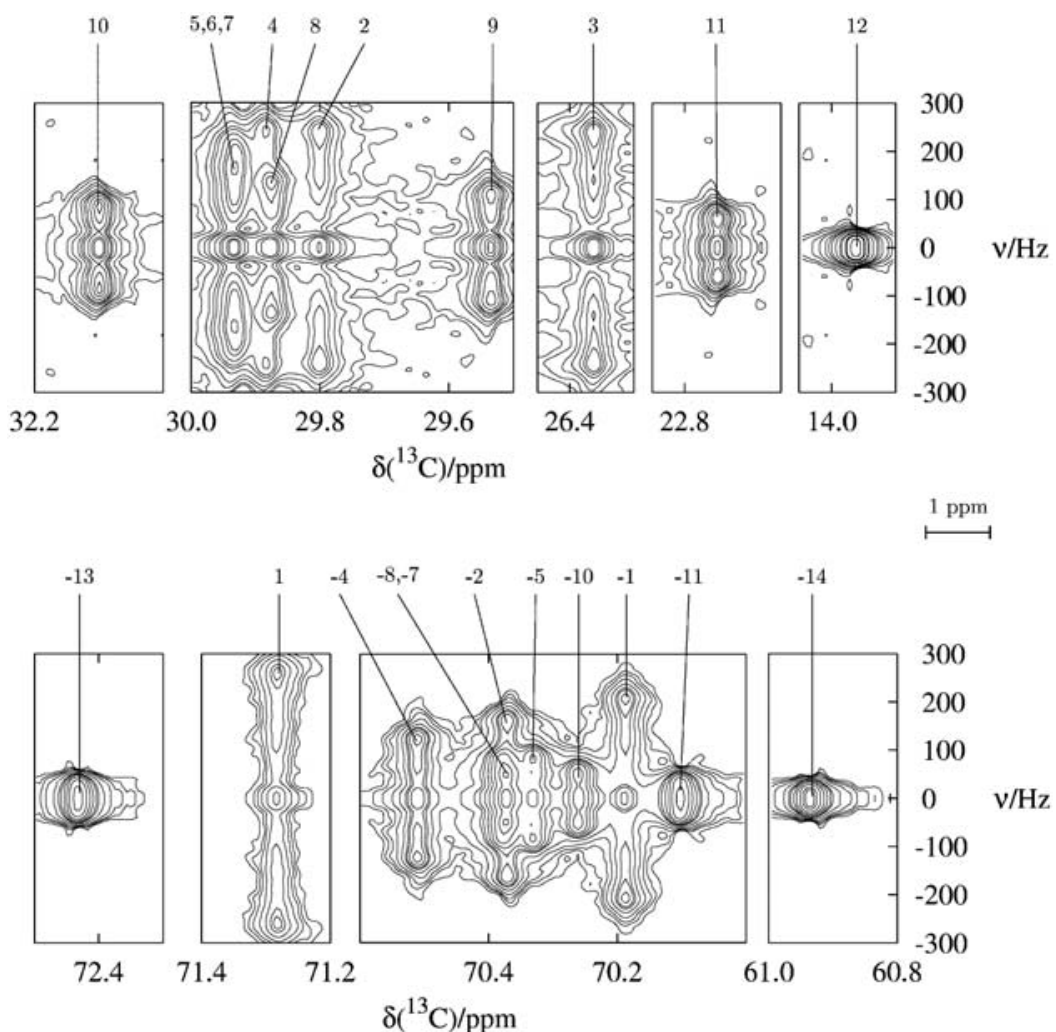

Fig. $52 \mathrm{D}{ }^{1} \mathrm{H}-{ }^{13} \mathrm{C}$ R-PDLF spectrum of a $\mathrm{C}_{12} \mathrm{E}_{5} / \mathrm{D}_{2} \mathrm{O}$ mixture with $57.7 \mathrm{wt} \% \mathrm{C}_{12} \mathrm{E}_{5}$ at $335 \mathrm{~K}$.

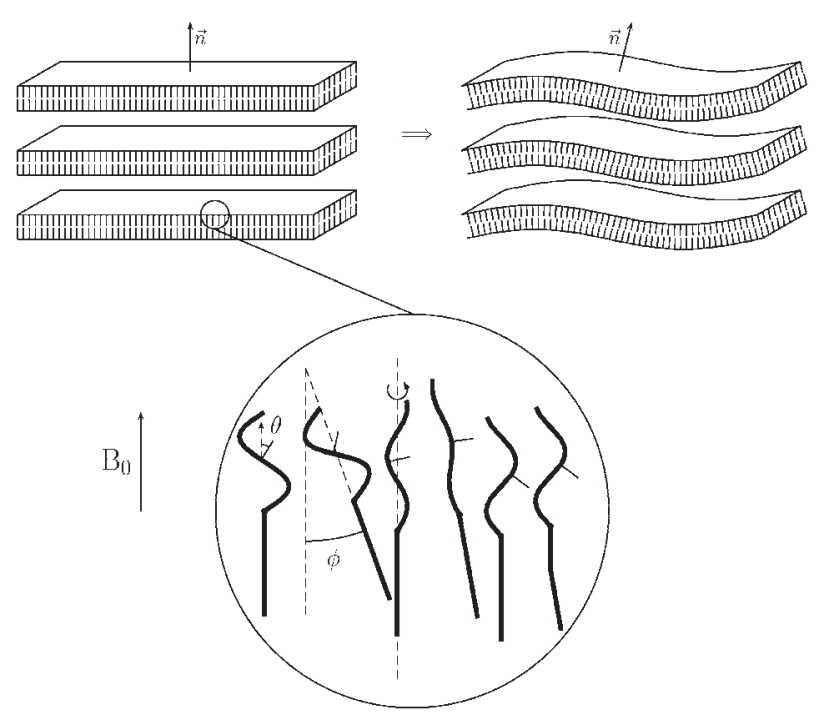

Fig. 6 Sketch illustrating the three types of orientational dependence of dipolar couplings referred to in the text. $\vec{n}$ represents the normal vector of the lamellar surface. $\phi$ is the tilting angle between the molecular axis and $\vec{n}$. It can be seen that $\theta$, the angle between the $\mathrm{C}-\mathrm{H}$ bond and the magnetic field, $\mathrm{B}_{0}$, is dependent on the direction of $\vec{n}, \phi$, and the conformation of the molecule. The arrow from left to right indicates the increase in lamellar undulation amplitude with temperature.

dipolar couplings with temperature provides information about conformational changes along the surfactant chain. These functions can be seen in Fig. 7.
Assuming that the ${ }^{13} \mathrm{C}-\mathrm{H}$ bonds of the $\alpha$ carbon (labeled 1 in Fig. 3) have minor deviations with respect to the long axis direction of the molecule, then one can think of the $\mathrm{C}_{\alpha}$ coupling magnitude as a measure of the lamella curvature. In fact, this assumption, combined with results obtained using other techniques, ${ }^{22}$ has been the basis of a large number of ${ }^{2} \mathrm{H}$ NMR studies, ${ }^{6,9,36}$ where the maximum of the $\mathrm{C}_{\alpha}$ quadrupolar coupling as function of temperature was interpreted as the transition of the lamellar phase, $\mathrm{L}_{\alpha}$, to a porous lamellar phase, $\mathrm{L}_{\alpha}^{H}$. As the curvature is related to the degree of hydration, then the $\mathrm{C}_{\alpha}$ splitting is mainly dependent on the fraction of hydrated EO units. Surprisingly, it was shown by Baciu et al. that the quadrupolar splitting from heavy water in a $\mathrm{C}_{12} \mathrm{E}_{5} / \mathrm{D}_{2} \mathrm{O}$ system, is directly proportional to the splitting obtained from an $\alpha$-deuterated surfactant. ${ }^{9}$

Experimental results ${ }^{8,37-39}$ as well as theoretical models, ${ }^{40,41}$ molecular dynamics ${ }^{42}$ and Monte Carlo simulations ${ }^{43}$ predicted that the lowest energy conformations for the-OCCOsegments correspond to a gauche conformation around the $\mathrm{C}-\mathrm{C}$ bond and anti around the $\mathrm{C}-\mathrm{O}$ bond. This is the dominant conformation at low temperature but with a low statistical weight over the complete set of possible conformations and it has the highest dipole moment of all conformers. ${ }^{44,45}$ Also, it has been shown that the fraction of EO units in the $\mathrm{C}-\mathrm{C}$ gauche conformation is dependent on the water content. $^{8,46}$ At higher temperature, conformations with low dipole moment become more populated as well as zero dipole moment trans conformations. This results in an increase of the order in the oxyethylene chains and the solute-solute interaction becomes more favorable than the solute-polar solvent 


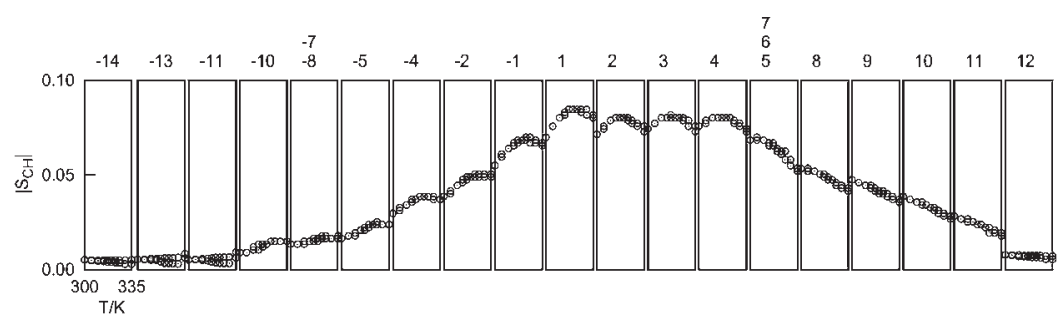

Fig. 7 Order parameters of the different $\mathrm{C}-\mathrm{H}$ bonds in $\mathrm{C}_{12} \mathrm{E}_{5} / \mathrm{D}_{2} \mathrm{O}$ (lamellar phase with $57 \mathrm{wt} \%$ of surfactant) as function of temperature. Measurements obtained in both increasing and decreasing temperature directions are plotted.

interaction. It is then straightforward to interpret the increase in the splitting of the oxyethylene carbons (Fig. 7) as being a consequence of the higher order proportioned by this change in the conformation distribution.

At higher temperatures, the decrease in splitting of the EO $\mathrm{C}-\mathrm{H}$ bonds is expected. The fast motion of the molecules and the increase in amplitude undulations of the lamellar phase ${ }^{47,48}$ oppose the effect of dehydration (Fig. 6). From a close inspection of the functions ( -10 to 5$)$, in Fig. 7 one can see that a maximum appears. Also, one can see a shift in the temperature value of the maximum, which decreases in the direction of the outer hydrophobic segments. The behavior of the hydrophobic tails with temperature is opposite to that of the headgroups, as can be seen in Fig. 7. At higher temperature the population of gauche conformations increases with the concomitant decrease of the order parameter. This suggests that the maximum in the $\alpha$ carbon, previously interpreted as being a consequence of a mesh phase, may have a simpler explanation, that is the compromise between the opposite trends of the EO and tail groups.

The balance between the contraction (trans $\rightarrow$ gauche) and extension (gauche $\rightarrow$ trans) of the tail and headgroup, respectively, with increasing temperature, agrees with the observation that the thickness of the lamella remains nearly constant with temperature. ${ }^{49}$ This conformational behavior is also observed in the chemical shifts, as, with increasing temperature, the chemical shifts of the headgroup carbons decrease while in the tail carbons an increase is observed (Fig. 4) as already had been pointed out by Ahlnäs et al. ${ }^{38}$

\section{Conclusion}

The R-PDLF method offers a complete $\mathrm{S}_{\mathrm{CH}}$ analysis of the $\mathrm{L}_{\alpha}$ surfactant liquid crystalline systems. The maximum observed for the $\alpha$-labeled $\mathrm{C}_{12} \mathrm{E}_{5}$ was previously interpreted as a mesh phase. A complete $\mathrm{S}_{\mathrm{CH}}$ profile suggests that the maximum observed for the central region may be a consequence of the opposing trends for the alkyl and oxyethylene parts.

\section{Acknowledgements}

Financial support from the Swedish Research Council (KFI/ VR) and the Swedish Foundation for Strategic Research (SSF). Bruno Medronho thanks Fundação de Ciência e Tecnologia (FCT) (project ref. SFRH/BD/21467/2005). R.W.M. acknowledges US National Institutes of Health (contract I ROI GM07825801). We gratefully acknowledge
Claudia Schmidt, Björn Lindman, Gunnar Karlström and Ulf Olsson for fruitful discussions.

\section{References}

1 P. Alexandridis and B. Lindman, Amphiphilic Block Copolymers (Self-Assembly and Applications), Elsevier Science BV, Amsterdam, 2000.

2 J. P. Douliez, A. M. Bellocq and E. J. Dufourc, J. Chem. Phys., 1994, 91, 874.

3 W. Schnepp and C. Schmidt, Ber. Bunsen-Ges. Phys. Chem., 1994, 98, 248.

4 M. Lukaschek, S. Müller, A. Hasenhindl, D. A. Grabowski and C. Schmidt, Colloid Polym. Sci., 1996, 274, 1.

5 F. Auguste, J. P. Douliez, A. M. Bellocq and E. J. Dufourc, Langmuir, 1997, 13, 666.

6 C. E. Fairhurst, M. C. Holmes and M. S. Leaver, Langmuir, 1997, 13, 4964.

7 S. Múller, C. Bórschig, W. Gronski, C. Schmidt and D. Roux, Langmuir, 1999, 15, 7558.

8 A. Tonegawa, K. Ohno, H. Matsuura, K. Yamada and T. Okuda, J. Phys. Chem. B, 2002, 106, 13211.

9 M. Baciu, U. Olsson, M. S. Leaver and M. C. Holmes, J. Phys. Chem. B, 2006, 110, 8184.

10 M. G. Munowitz, R. G. Griffin, G. Bodenhausen and T. H. Huang, J. Am. Chem. Soc., 1981, 103, 2529.

11 D. P. Weitekamp, J. R. Garbow and A. Pines, J. Chem. Phys., 1982, 77(6), 2870.

12 Y. K. Lee, N. D. Kurur, M. Helme, O. G. Johannessen, N. C. Nielsen and M. H. Levitt, Chem. Phys. Lett., 1995, 242, 304.

13 J. D. Gross, D. E. Warchawski and R. G. Griffin, J. Am. Chem. Soc., 1997, 119, 796.

14 M. Caravetta, M. Edén, X. Zhao, A. Brinkmann and M. H. Levitt, Chem. Phys. Lett., 2000, 321, 205.

15 X. Zhao, M. Edén and M. H. Levitt, Chem. Phys. Lett., 2001, 342, 353.

16 S. V. Dvinskikh, H. Zimmermann, A. Maliniak and D. Sandström, J. Magn. Reson., 2004, 168, 194.

17 A. E. Bennet, R. G. Griffin and S. Vega, in NMR Basic Principles and Progress, Springer-Verlag, Berlin Heidelberg, 1994, vol. 33, pp. 3-77.

18 M. H. Levitt, J. Chem. Phys., 2008, DOI: 10.1063/1.2831927.

19 S. V. Dvinskikh, V. Castro and D. Sandström, Phys. Chem. Chem. Phys., 2005, 7, 607.

20 V. Castro, S. V. Dvinskikh, G. Widmalm, D. Sandström and A. Maliniak, Biochim. Biophys. Acta, 2007, 1768, 2432.

21 C. Leal, D. Sandström, P. Nevsten and D. Topgaard, Biochim. Biophys. Acta, 2008, 1778, 214.

22 M. Imai, A. Saeki, T. Teramoto, A. Kawaguchi, K. Nakaya, T. Kato and K. Ito, J. Chem. Phys., 2001, 115, 10525.

23 D. D. Laws, H. L. Bitter and A. Jerschow, Angew. Chem., Int. Ed., 2002, 41, 3096.

24 T. G. Oas, R. G. Griffin and M. H. Levitt, J. Chem. Phys., 1988, 89, 1988.

25 T. Gullion and J. Schaefer, J. Magn. Reson., 1989, 81, 196.

26 J. C. C. Chan and H. Eckert, J. Chem. Phys., 2001, 115, 6095.

27 M. H. Levitt, in Encyclopedia of Nuclear Magnetic Resonance: Supplementary Volume, ed. D. M. Grant and R. K. Harris, Wiley, Chichester, UK, 2002. 
28 X. Zhao, W. Hoffbauer, J. S. auf der Günne and M. H. Levitt, Solid State Nucl. Magn. Reson., 2004, 26, 57.

29 G. A. Morris and R. Freeman, J. Am. Chem. Soc., 1979, 101, 760.

30 J. D. Gross, P. R. Costa, J. P. Dubacq, D. E. Warchawski, P. N. Lirsac, P. F. Devaux and R. G. Griffin, J. Magn. Reson., Ser. B, 1995, 106, 187.

31 A. E. Bennet, C. M. Rienstra, M. Auger, K. V. Lakshmi and R. G. Griffin, J. Chem. Phys., 1995, 103, 6051

32 A. L. V. Geet, Anal. Chem., 1970, 42, 679.

33 L. Zheng, M. Suzuki, T. Inoue and B. Lindman, Langmuir, 2002, 18, 9204.

34 A. A. Ribeiro and E. A. Dennis, J. Phys. Chem., 1977, 81, 957.

35 P. Quist, B. Halle and I. Furó, J. Chem. Phys., 1991, 95(9), 6945.

36 B. Medronho, M. G. Miguel and U. Olsson, Langmuir, 2007, 23, 5270.

37 V. Viti and P. Zampetti, Chem. Phys., 1973, 2(2), 233.

38 T. Ahlnäs, G. Karlström and B. Lindman, J. Phys. Chem., 1987, 91, 4030 .
39 S. A. Wahab and H. Matsuura, Chem. Lett., 2001, 3, 198.

40 M. Andersson and G. Karlström, J. Phys. Chem., 1985, 89 4957.

41 G. Karlström, J. Phys. Chem., 1985, 89, 4962.

42 S. Bandyopadhyay, M. Tarek, M. L. Lynch and M. L. Klein, Langmuir, 2000, 16, 942.

43 Y. C. Kong, D. N. N. G. Parsonage and L. Thompson, J. Chem. Soc., Faraday Trans., 1994, 90(16), 2375.

44 B. Lindman, A. Carlsson, G. Karlström and M. Malmsten, $A d v$. Colloid Interface Sci., 1990, 32, 183.

45 G. Karlström and B. Lindman, in Organized Solutions, ed. S. E. Friberg and B. Lindman, Marcel Deker, Inc., New York, 1992, pp. 49-65.

46 O. Engkvist and G. Karlström, J. Phys. Chem. B, 1997, 101, 1631. 47 W. Helfrich, Z. Naturforsch., 1978, 33a, 305.

48 H. Bagger-Jörgensen and U. Olsson, Langmuir, 1996, 12, 4057.

49 K. Minewaki, T. Kato, H. Yoshida, M. Imai and K. Ito, Langmuir, 2001, 17, 1864. 